\title{
Special issue: advanced techniques for radar signal processing
}

\author{
D. Orlando ${ }^{1 *}$, C. Hao ${ }^{2}$, A. Aubry ${ }^{3}$, G. Cui ${ }^{4}$, A. C. Gurbuz ${ }^{6}$ and S. Gazor ${ }^{5}$
}

Recent advances in technology have led to the development of low-cost sensing devices capable of providing high performances in terms of both computational resources and measurement precision. More important, the reduced size of such devices have allowed to exploit them in diverse application fields (as, for instance, medical, military, manufacturing, transportation, and safety systems). However, in the context of military applications (e.g., radar and communication systems), the downside of this technology is that it is available for terrorist attacks aimed at denying targeting information and using radarguided missiles or small drones carrying dangerous (e.g., explosive or chemical) substances. Thus, it stems the need for innovative signal processing solutions to counteract these threats. Such techniques are applicable in ship and aircraft monitoring (for defense purposes), coastal surveillance, and, generally speaking, homeland security.

This special issue focuses on radar signal processing techniques (target detection and tracking, interference estimation and suppression, adaptive beamforming, electronic warfare) that benefit from the mentioned advances to face the new challenging operating scenarios that naturally arise from nowadays technology advantages and disadvantages. More specifically, the emphasis is on (possibly distributed) radar systems equipped with arrays of sensors, which enable to capitalize the spatial diversity and power integration enabling significant improvements in performance.

In general, radar systems perform three general functions, which are search, track, and imaging. The most important operation of a search radar is target detection. As a matter of fact, once the system declares the presence of a target, its resources are scheduled to estimate target parameters [1,2] or for target tracking [3] which consists in the fine estimation of parameters as range, azimuth angle, elevation angle, and Doppler frequency offset.

*Correspondence: danilo.orlando@unicusano.it

'Università degli Studi "Niccolò Cusano", via Don Carlo Gnocchi 3, 00166 Roma, Italy

Full list of author information is available at the end of the article
Moreover, it is worth underlining that range resolution can be increased by means of pulse compression techniques which exploit coded signals $[4,5]$.

Thus, it is clear that the reduction and/or the control of the number of false alarms, which waste hardware resources, is of primary concern in radar signal processing. The main sources generating false targets are clutter (unwanted echoes from the environment), thermal noise, and intentional interferences also known as electronic countermeasures (ECM) (coherent and/or noise-like jammers). In this respect, several contributions presented here are aimed at mitigating the effects of these interfering signals [6-11]. Some of these describe algorithms which clean acquired data from intentional interference components and feed the following detection algorithms, whereas other papers devise detection algorithms providing the so-called constant false alarm rate (CFAR) property with respect to the clutter and thermal noise $[9,12]$. The former techniques are classified as electronic counter-countermeasures (ECCM) (see also [13-17]). Further contributions deal with distributed systems (bistatic, multistatic, or sensor networks) [10, 12], which, due to the additional number of degrees of freedom, are more effective against the deleterious effects due to false alarms, stealth targets, shadowing, multipath, and intentional or unintentional interference. Finally, waveform design and compressive sensing techniques applied to MIMO radars [18-20] and signal reconstruction techniques [21-23] are also considered here.

Once a target is detected in track, the imaging function comes in handy for target classification, discrimination, and/or identification purposes. As a matter of fact, it provides high-resolution data in range, azimuth, elevation, and sometimes Doppler. A well-known example of imaging radar is represented by the Synthetic Aperture Radar (SAR), which provides a fine-resolution two-dimensional intensity image of the illuminated scene. SAR images can be exploited for mapping and land-use surveying, detection, location, and identification of fixed targets [24-31]. Some of the above aspects are considered in several contributions collected here. Another delicate issue related 
to SAR systems is the image focalization which is made difficult by undesired platform movements. Techniques aimed at the compensation of these effects are herein considered also in the context of compressive processing $[24,25,28,29]$.

The final topic addressed in this special issue concerns the electronic warfare and in particular signal processing techniques for electronic-signals intelligence [32,33].

\section{Competing interests}

The authors declare that they have no competing interests.

\section{Publisher's Note}

Springer Nature remains neutral with regard to jurisdictional claims in published maps and institutional affiliations.

\section{Author details \\ ${ }^{1}$ Università degli Studi "Niccolò Cusano", via Don Carlo Gnocchi 3, 00166 Roma, Italy. ${ }^{2}$ Institute of Acoustics, Chinese Academy of Sciences, 100190 Beijing, China. ${ }^{3}$ Dipartimento di Ingegneria Elettrica e delle Tecnologie dell'Informazione, Università degli Studi di Napoli Federico II, via Claudio 21, 80125 Napoli, Italy. ${ }^{4}$ School of Electronic Engineering, University of Electronic Science and Technology of China, Chengdu, China. ${ }^{5}$ Department of Electrical and Computer Engineering, Walter Light Hall, Queen's University, Kingston, Ontario, Canada. ${ }^{6}$ TOBB University of Economics and Technology, Ankara, Turkey.}

Received: 15 May 2017 Accepted: 23 May 2017

Published online: 28 June 2017

\section{References}

1. B Wang, YD Zhang, W Wang, Robust group compressive sensing for DOA estimation with partially distorted observations. EURASIP Journal on Advances in Signal Processing, Special Issue on Advanced Techniques for Radar Signal Processing

2. JY Liu, YL Lu, YM Zhang, WJ Wang, Fractional difference co-array perspective for wideband signal DOA estimation. EURASIP Journal on Advances in Signal Processing, Special Issue on Advanced Techniques for Radar Signal Processing

3. LW Jochumsen, J Ostergaard, SH Jensen, C Clemente, MO Pedersen, A recursive kinematic random forest and alpha beta filter classifier for $2 \mathrm{D}$ radar tracks. EURASIP Journal on Advances in Signal Processing, Special Issue on Advanced Techniques for Radar Signal Processing

4. A Hanif, AB Mansoor, AS Imran, Sub-Nyquist sampling and detection in Costas coded pulse compression radars. EURASIP Journal on Advances in Signal Processing, Special Issue on Advanced Techniques for Radar Signal Processing

5. X Yu, G Cui, D Ran, Fast implementation for modified adaptive multi-pulse compression. EURASIP Journal on Advances in Signal Processing, Special Issue on Advanced Techniques for Radar Signal Processing

6. Y Wang, Q Bao, Z Chen, Robust mainlobe interference suppression for coherent interference environment. EURASIP Journal on Advances in Signal Processing, Special Issue on Advanced Techniques for Radar Signal Processing

7. XWang, J Xie, Z He, H Li, A Robust generalized sidelobe canceller via steering vector estimation. EURASIP Journal on Advances in Signal Processing, Special Issue on Advanced Techniques for Radar Signal Processing

8. T Liu, R Min, Y Pi, K Long, Z Huang, Binary integration nonparametric detection for range-spread targets in distributed terahertz radar network under unknown clutter. EURASIP Journal on Advances in Signal Processing, Special Issue on Advanced Techniques for Radar Signal Processing

9. S Fortunati, F Gini, MS Greco, Matched, mismatched and robust scatter matrix estimation and hypothesis testing in complex t-distributed data. EURASIP Journal on Advances in Signal Processing, Special Issue on Advanced Techniques for Radar Signal Processing
10. F Ansari, MR Taban, S Gazor, A Novel sequential algorithm for clutter and direct signal cancellation in passive bistatic radars. EURASIP Journal on Advances in Signal Processing, Special Issue on Advanced Techniques for Radar Signal Processing

11. J Qian, Z He, J Xie, Y Zhang, Null broadening adaptive beamforming based on covariance matrix reconstruction and similarity constraint. EURASIP Journal on Advances in Signal Processing, Special Issue on Advanced Techniques for Radar Signal Processing

12. R Palamà, M Greco, F Gini, Multistatic adaptive CFAR detection in non-Gaussian clutter. EURASIP Journal on Advances in Signal Processing, Special Issue on Advanced Techniques for Radar Signal Processing

13. A Abdalla, W Wang, Z Yuan, S Mohamed, T Bin, Subarray-based FDA radar to counteract deceptive ECM signals. EURASIP Journal on Advances in Signal Processing, Special Issue on Advanced Techniques for Radar Signal Processing

14. C Shi, S Salous, F Wang, J Zhou, Low probability of intercept based adaptive radar waveform optimization in signal-dependent clutter for joint radar and cellular communication systems. EURASIP Journal on Advances in Signal Processing, Special Issue on Advanced Techniques for Radar Signal Processing

15. W Xiong, G Zhang, W Liu, Efficient filter design against interrupted sampling repeater jamming for wideband radar. EURASIP Journal on Advances in Signal Processing, Special Issue on Advanced Techniques for Radar Signal Processing

16. Z Wang, W Wang, H Shao, Range-azimuth decouple beamforming for frequency diverse array with Costas-sequence modulated frequency offsets. EURASIP Journal on Advances in Signal Processing, Special Issue on Advanced Techniques for Radar Signal Processing

17. $\mathrm{P} \mathrm{Ge}, \mathrm{G}$ Cui, L Kong, J Yang, Unimodular sequence design under frequency hopping communication compatibility requirements. EURASIP Journal on Advances in Signal Processing, Special Issue on Advanced Techniques for Radar Signal Processing

18. X Yu, G Cui, M Piezzo, S lommelli, L Kong, Robust constrained waveform design for MIMO radar with uncertain steering vectors. EURASIP Journal on Advances in Signal Processing, Special Issue on Advanced Techniques for Radar Signal Processing

19. N Shahbazi, A Abbasfar, M Jabbarian-Jahromi, Efficient two-dimensional compressive sensing in MIMO radar. EURASIP Journal on Advances in Signal Processing, Special Issue on Advanced Techniques for Radar Signal Processing

20. H Ali, S Ahmed, TY Al-Naffouri, MS Sharawi, M Alouini, Target parameter estimation for spatial and temporal formulations in MIMO Radars using Compressive Sensing. EURASIP Journal on Advances in Signal Processing, Special Issue on Advanced Techniques for Radar Signal Processing

21. K Jiang, S Chen, B Tang, The RIP and block-RIP analysis of Nyquist folding receiver for recovering signals. EURASIP Journal on Advances in Signal Processing, Special Issue on Advanced Techniques for Radar Signal Processing

22. D Zhang, W Wu, D Fang, Array signal recovery algorithm for a single-RFchannel DBF array. EURAJournal, SIP, on Advances in Signal Processing, Special Issue on Advanced Techniques for Radar Signal Processing

23. K Jiang, I Zhu, B Tang, Spectral reconstruction of signals from periodic nonuniformly subsampling based on a Nyquist folding scheme. EURASIP Journal on Advances in Signal Processing, Special Issue on Advanced Techniques for Radar Signal Processing

24. WXia, L Huang, Target vibration estimation in SAR based on phaseanalysis method. EURASIP Journal on Advances in Signal Processing, Special Issue on Advanced Techniques for Radar Signal Processing

25. $\mathrm{W} \mathrm{Pu}, \mathrm{Y}$ Huang, J Wu, J Yang, $\mathrm{W} \mathrm{Li}$, A residual range cell migration correction algorithm for bistatic forward-looking SAR. EURASIP Journal on Advances in Signal Processing, Special Issue on Advanced Techniques for Radar Signal Processing

26. $\mathrm{S} \mathrm{Liu,} \mathrm{Z} \mathrm{Cao,} \mathrm{H} \mathrm{Wu,} \mathrm{Y} \mathrm{Pi,} \mathrm{H} \mathrm{Yang,} \mathrm{Target} \mathrm{detection} \mathrm{in} \mathrm{complex} \mathrm{scene} \mathrm{of} \mathrm{SAR}$ image based on existence probability. EURASIP Journal on Advances in Signal Processing, Special Issue on Advanced Techniques for Radar Signal Processing

27. JY Baudais, S Méric, V Riché, É Pottier, MIMO-OFDM signal optimization for SAR imaging radar. EURASIP Journal on Advances in Signal Processing, Special Issue on Advanced Techniques for Radar Signal Processing

28. L Zhang, H Wang, Z Qiao, Resolution enhancement for ISAR imaging via improved statistical compressive sensing. EURASIP Journal on Advances 
in Signal Processing, Special Issue on Advanced Techniques for Radar Signal Processing

29. G Wang, L Zhang, J Li, Q Hu, Range cell migration correction analysis of one-step and two-step motion compensation for millimeter-wave airborne SAR imaging. EURASIP Journal on Advances in Signal Processing, Special Issue on Advanced Techniques for Radar Signal Processing

30. NO Onhon, M Cetin, SAR moving object imaging using sparsity imposing priors. EURASIP Journal on Advances in Signal Processing, Special Issue on Advanced Techniques for Radar Signal Processing

31. CWang, X Wena, $\mathrm{H}$ Xua, A robust estimator of parameters for $\mathrm{G}$-modelled SAR imagery based on random weighting method. EURASIP Journal on Advances in Signal Processing, Special Issue on Advanced Techniques for Radar Signal Processing

32. W Pei, Q Zhaoyang, Z Jun, T Bin, Autonomous radar pulse modulation classification using modulation components analysis. EURASIP Journal on Advances in Signal Processing, Special Issue on Advanced Techniques for Radar Signal Processing

33. Q Zhaoyang, W Pei, Z Jun, T Bin, A parameter estimation algorithm for LFM/BPSK hybrid modulated signal intercepted by Nyquist folding receiver. EURASIP Journal on Advances in Signal Processing, Special Issue on Advanced Techniques for Radar Signal Processing

\section{Submit your manuscript to a SpringerOpen ${ }^{\circ}$ journal and benefit from:}

- Convenient online submission

- Rigorous peer review

- Open access: articles freely available online

- High visibility within the field

- Retaining the copyright to your article

Submit your next manuscript at $\gg$ springeropen.com 